\title{
Surface Roughness Image Analysis using Quasi-Fractal Characteristics and Fuzzy Clustering Methods
}

\author{
Tiberiu Vesselenyi, Ioan Dzitac, Simona Dzitac, Victor Vaida
}

\begin{abstract}
In this paper the authors describe the results of experiments for surface roughness image acquisition and processing in order to develop an automated roughness control system. This implies the finding of a characteristic roughness parameter (for example $\mathrm{Ra}$ ) on the bases of information contained in the image of the surface. To achieve this goal we use quasi-fractal characteristics and fuzzy clustering methods.
\end{abstract}

Keywords: image processing, surface roughness, quasi- fractal parameters, fuzzy clustering.

\section{Introduction}

Surface roughness of manufactured products is defined in SR ISO 4287/2001 standard and other international standards. Simple and complex characterization parameters are explained in works like [1], which are considering the use of stylus devices to measure roughness after a linear or curved path $[3,7,7,12]$. Although these devices had been continuously upgraded in order to increase measuring precision [3], they are not efficient enough used in automated measuring systems, due to the fact that the stylus must make contact with the measured surface and also due to the very long time of measurement. A newer technique in surface roughness measurement is the employment of digital image acquisition and processing $[4,6]$. In this case the camera is coupled to a microscope (bellow a magnification of $x 100$ ) and the acquisitioned images are processed with specially designed computer programs. So one image of $24 \mathrm{~mm}^{2}$, corresponds to 100 stylus scanning.

In the paper [10] a method of summit and directionality identification of textured images is defined using surface image analysis. In [2] there is shown that surfaces obtained by turning, milling and grinding presents a high complexity when they are analyzed by optical, electron microscopes or AFM (Atomic Force Microscope). Isotropic surfaces obtained by machining can be characterized by the MandelbrotWeierstrass function using fractal methods. For the characterization of anisotropic surfaces some authors are proposing methods based on the two dimensional FFT algorithm. This method had been used for characterization of grinded surface.

The basic idea in [11] is to decompose the surface roughness (described in terms of amplitude, wave length and direction) in convex elements (summits) and then analyze these structures with morphologic trees. The authors claim that clear correlations can be obtained between the obtained morphologic tress and the tribologic proprieties of the surfaces.

In [4] the correspondence between surface roughness obtained with a certain manufacturing method and its image had been studied based on functional dependence between roughness height and the grey level values of the image. The authors had studied these correspondences with the help of polynomial nets, trained with experimental results, which has as inputs cutting speed, feed and cutting depth and also the mean values of image grey levels. The polynomial net is capable to estimate the surface roughness with an acceptable accuracy, which had been validated with a series of experimental measurements. The advantage of this method is the possibility to apply it to online roughness estimation in turning processes.

The paper [9] proposes a new method to analyze and characterize the surface roughness. On the basis of an algorithm in three steps the classification of textured images of some manufactured surfaces is made, surfaces obtained by casting, milling and grinding. In the first step the image is processed by a frequency normalized wavelet transform, obtaining a set of images at different scales and phases. In the 
second step characteristic parameter values are extracted and in the third step the image classification is obtained using the set of extracted features.

From the synthesis of presented works, it can be concluded that the method of surface image processing is better than the methods using the stylus type measurement, because it is faster and there is no contact between the measuring instrument and the surface. It also has been shown that there is a correlation between the height of the roughness and the image grey levels and the estimation of surface roughness can be done in similar ways as texture analysis.

\section{Acquisition and Preprocessing of Surface Images}

For image acquisition purposes several manufactured roughness probes with known roughness parameters were used (STALÎ DOVODCA - GOST 9378-80 E15718) obtained by manufacturing operations as: cylindrical milling, plane milling, shaping, frontal grinding, plane grinding and polishing. In this paper only surface roughness images representing shaping, plane grinding and polishing will be analyzed, because these images are more similar to each other and harder to classify. Four non-overlapping images, of every probe's surface were taken using a CCD camera mounted on a CITIVAL microscope at magnifications of $\times 10$ and $\times 25$. The resolution of the images was $640 \times 480$ pixel. The correlation between surface roughness and surface image had been studied in a large number of papers [4] showing a certain functional dependency between asperity height and image intensity. During experiments however, we observed that this correlation is more complex and depends in a very high degree on the illumination conditions of the probe. Usual image processing phases, of non-object representation images are presented in [14].

After the image acquisition, a number of preprocessing operations had to be made in order to obtain better image quality. The used preprocessing steps were as follows:

- filtering - eliminate inherent image noises;

- establishing region of interest - keep only high information regions of the image;

- uneven illumination effects elimination - eliminate effects of higher intensity in the middle of the image, which is characteristic for images taken with microscopes (figure 1 and 2);

- correction of probe rotation and position variations - as the images are anisotropic, rotation of the probe can alter the analysis results. Here 2D Fast Fourier Transforms described in [2] or oriented Gabor filters described in [5], can be used. The authors had tested several automatic image rotation algorithms and finally a 1D FFT method was used.

On the base of tested preprocessing methods a program module had been developed, which can perform al preprocessing steps automatically and which can be eventually included in an automated quality control system. After preprocessing, the image quality was fair enough to perform the next step of image processing. Studying recent researches in texture analysis and image processing a number of statistical methods (co-occurrence, statistical moments) and frequency domain methods (Gabor filters, wavelet analysis), had been tested in order to obtain automated recognition of surface roughness parameters, but these methods didn't yield the wanted results. So we focused our research on fractal methods.

\section{Fractal Image Processing Characterization}

Considering the goal of our research, computation of fractal dimension is less important from a practical point of view. It is more important to use fractal or pseudo-fractal parameters in order to discriminate surfaces with different roughness characteristics. Fractal dimension computation of rough 

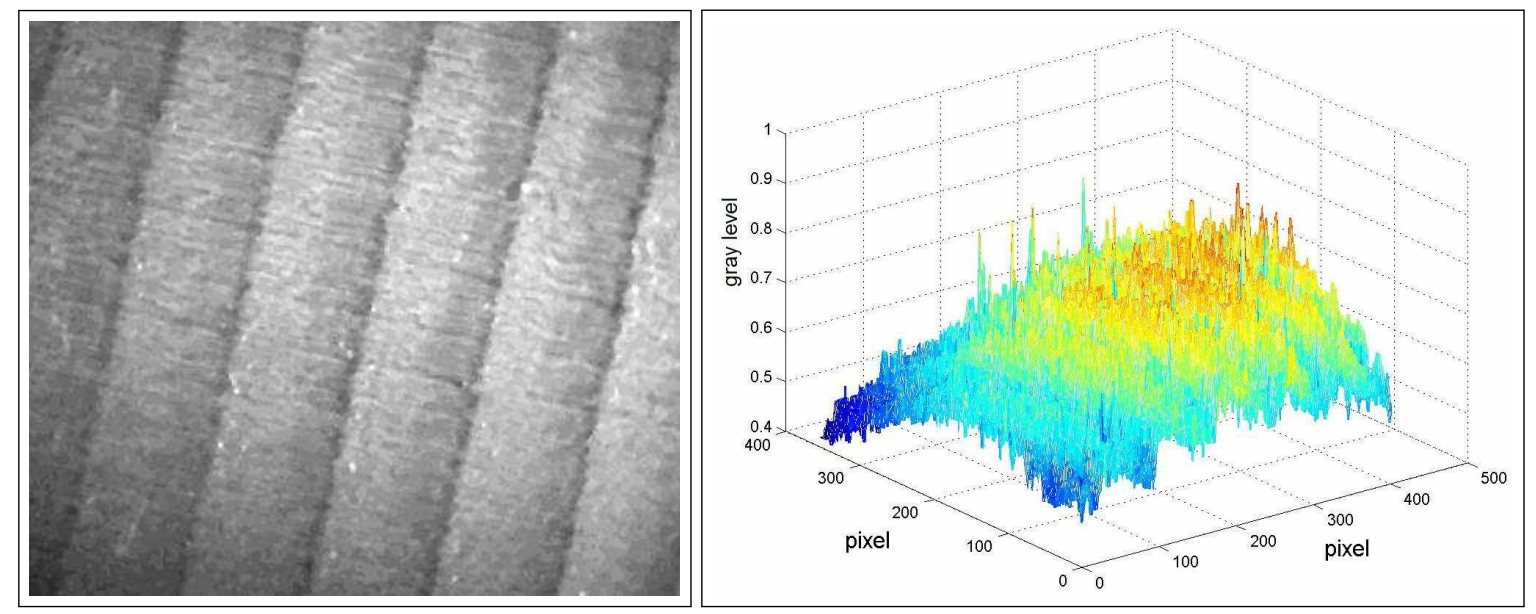

Figure 1: Surface image with higher center intensity.
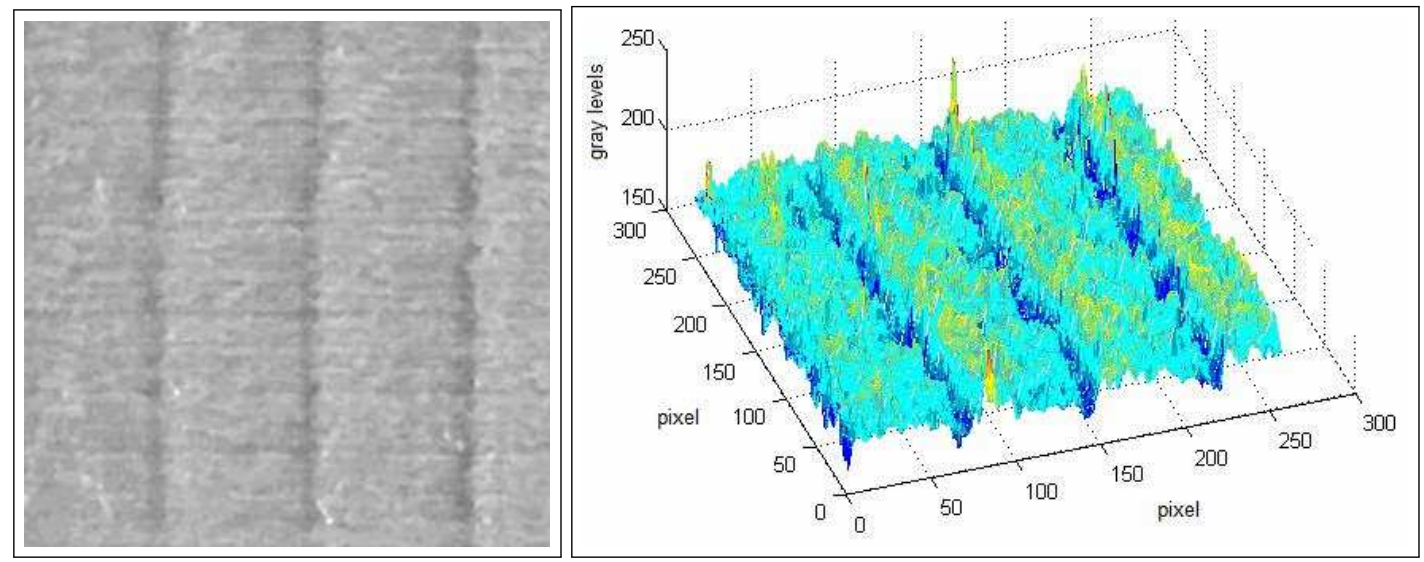

Figure 2: Image after preprocessing (uneven lighting and rotation has been eliminated). 
surfaces using the Weierstrass-Mandelbrot function is described in [2] and others. When this function is correlated to power spectral density, the fractal dimension is correlated to the slope of the spectrum represented in logarithmic scale. The Weierstrass-Mandelbrot function is difficult to apply in practice. That is why we had to use methods, which are easier to implement as computer algorithms. These methods are the box counting method (BC) and the frequency domain fractal parameter (using power spectral density diagrams). Both methods had been tested on the roughness probes images. The box counting method (BC) had been derived from the "compass dimension" and is closely related to fractal dimension as it has been stated by Mandelbrot with the relation:

$$
D=\frac{\log N}{\log (1 / r)}
$$

The compass dimension is obtained measuring a curve (which can represent a section through a surface) with decreasing measuring units $\left(r_{i}, i=k \ldots 1\right)$ and storing the number of measures $N_{i}$ for each $r_{i}$. The diagram of $\log \left(N_{i}\right)$ as function of $\log \left(1 / r_{i}\right)$ is drawn obtaining a so called Richardson plot. If the Richardson plot is a straight line then the measured object is fractal and the slope of the plot is it's compass dimension. The BC method uses rectangular boxes of decreasing edges instead the linear measure $r_{i}$. Fractal dimension can also be computed on the bases of power spectral density (PSD), as it is stated in [1]. If the PSD amplitude is represented as a function of spatial frequency (f) in a logarithmic diagram then the fractal parameter can be considered as the slope $\left(p_{1}^{\prime}\right)$ of the $\log (\mathrm{PSD})$ approximation line and the $\left(p_{2}{ }^{\prime}\right)$ as the intersection of this line with the ordinate axis.

$$
\log (P S D)=p_{1}^{\prime} \log (f)+p_{2}^{\prime}
$$

First we have developed a 2D box counting algorithm and then a 3D algorithm (which uses 3D boxes on 3D matrix as shown in figure 2) both yielding satisfactory results. Although the obtained results show that the analyzed images do not have true fractal behavior (the resulted Richardson plot is not a rigorously straight line), the goal is to find correlations between obtained parameters on one hand and the surface roughness on the other hand.

In this research we had used the BC3D (3 dimensional box counting) method, but in the Richardson plot, instead of using only linear approximation in order to define parameters, we also used second and third degree polynomials. If we denote $y=\log \left(N_{i}\right)$ and $x=\log \left(1 / r_{i}\right)$, we will have the following relations:

$$
\begin{aligned}
y & =p_{1} x+p_{2} \\
y & =p_{3} x^{2}+p_{4} x+p_{5} \\
y & =p_{6} x^{3}+p_{7} x^{2}+p_{8} x+p_{9}
\end{aligned}
$$

Examples of curve fitting for relations (3), (4), (5) are given in figures 3, 4 and 5.

We had considered then to use coefficients p1Ěp9 to characterize the roughness of the studied manufactured surfaces. These coefficients had been named by us "quasi fractal parameters" (QFP, meaning fractal-like parameters) highlighting the fact that they are not rigorously correlated to fractal dimension.

In order to study the possibilities to classify images representing rough surfaces, with the above defined parameters, we have made two dimensional representations that we denoted as QFP diagrams (Quasi Fractal Parameter Diagrams). Each of these diagrams represents a bi-dimensional space having as dimensions two of the QFPs defined above. 


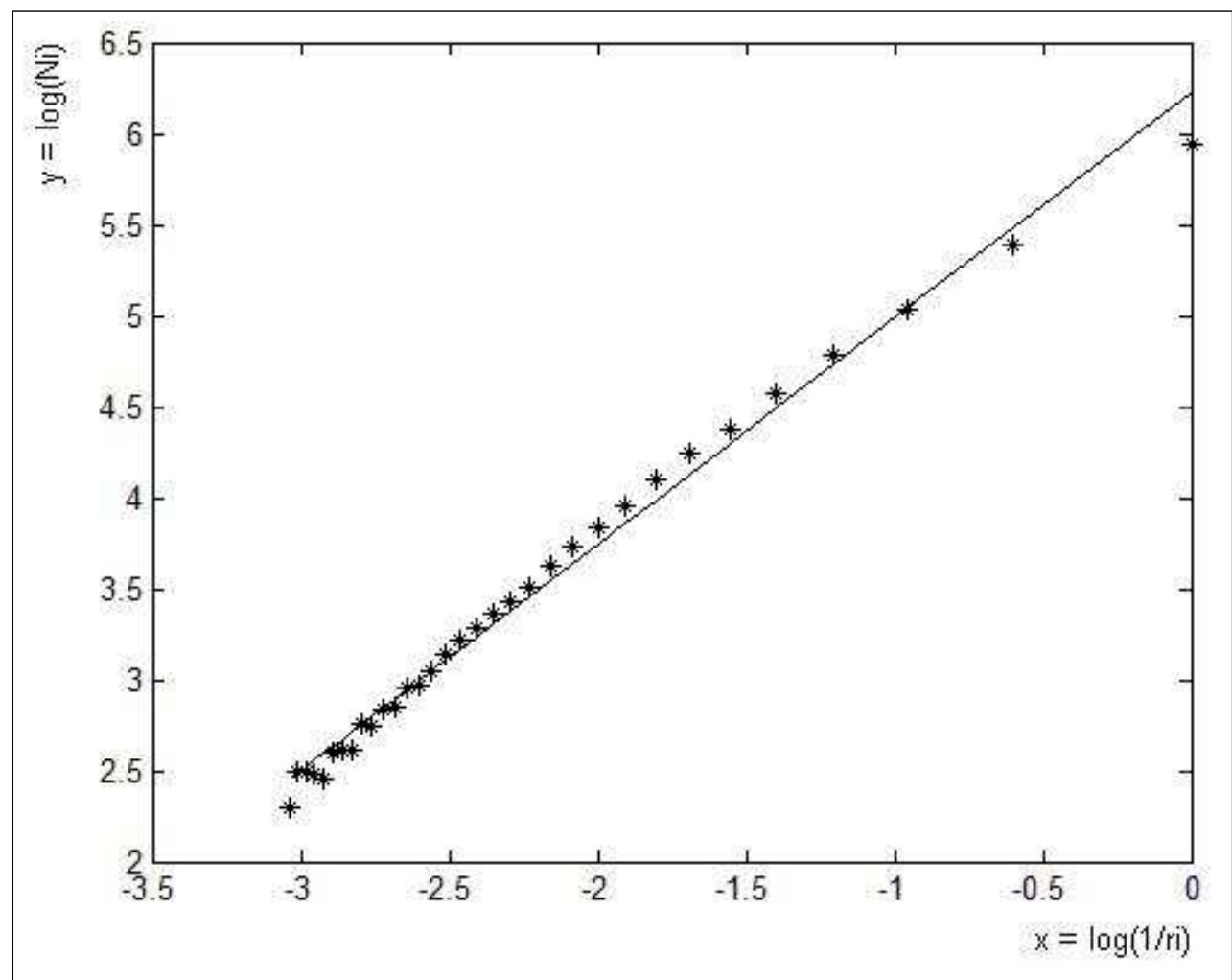

Figure 3: Linear curve fitting for points obtained with the BC3D algorithm applied on an image of shaped surface.

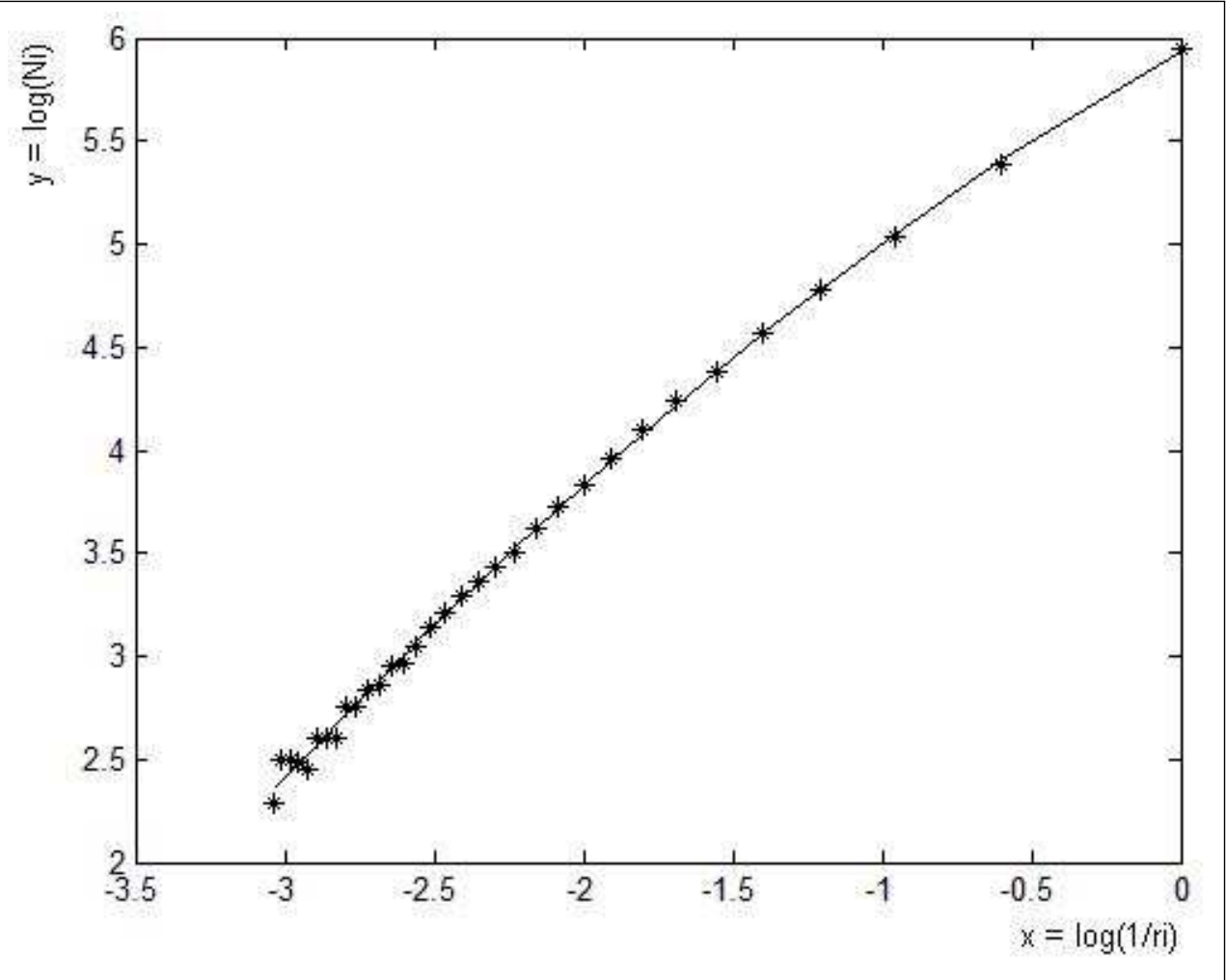

Figure 4: Second order polynomial curve fitting for points obtained with the BC3D algorithm applied on an image of shaped surface. 


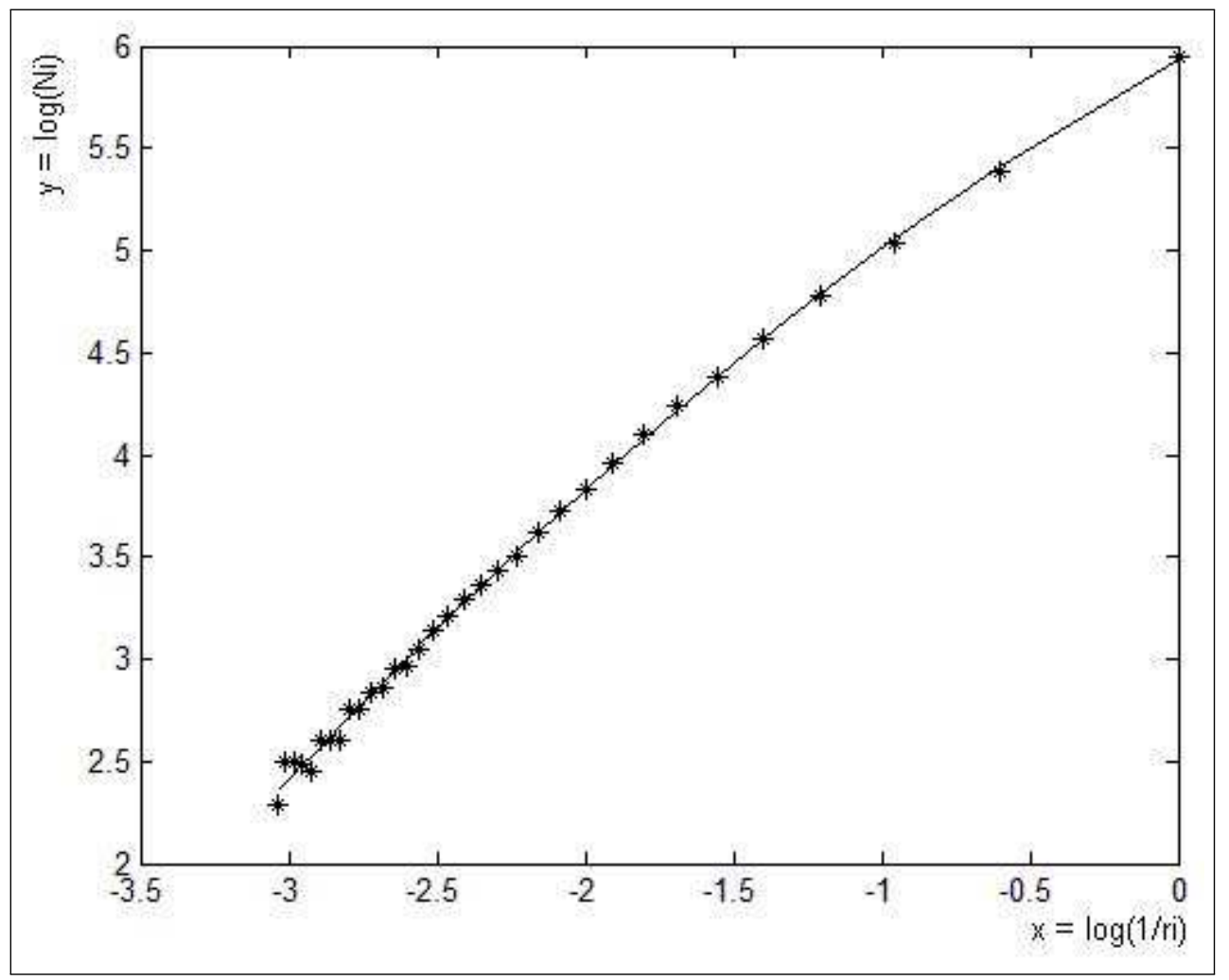

Figure 5: Third order polynomial curve fitting for points obtained with the BC3D algorithm applied on an image of shaped surface.

\section{Experimental Considerations}

In order to establish how QFPs can characterize surface roughness we had acquired and processed four different images from each surface roughness probe, for three types of cutting operations: shaping with 4 different roughness values, grinding with four different roughness values and polishing with three different roughness values. Table 1 shows sample categories, roughness values, sample codes and diagram symbols. In diagram symbols the color represents the operation and the symbol represents a specific roughness value.

Some examples of analyzed images are shown in figure 6.

There had been also acquired images for magnification of $\mathrm{x} 25$. For both magnifications, the images had been studied using QFP diagrams. Two examples of such diagrams are shown in figure 6.a and 6.b.

Comparing diagrams in figures 6.a. and 6.b., it can be observed that some of the same studied samples have different locations in the parameter space. For example the black squares (representing S4 samples) have one location for $\mathrm{x} 10$ magnification and other location for $\mathrm{x} 25$ magnification. This shows that $\mathrm{p} 1$ and $\mathrm{p} 2$ parameters are not "true fractal" parameters because they do not exhibit an invariance to scale, but this also shows that if in X10 magnification we can not really distinguish the S4 samples from the $\mathrm{G}$ group samples, at $\mathrm{x} 25$ magnification the $\mathrm{S} 4$ samples are well distinguishable. This lead to the conclusion that acquiring and analyzing sample images at different magnifications can help to better discriminate the samples. The selection of optimum magnifications to be used needs to be experimented for large sets of images. In this paper we will present discrimination algorithms only for samples with magnification of $x 10$. The establishing of box dimension range is also an issue, but this can be also solved with experiments on large sets of calibration images. The authors plan to present algorithms for these issues in a future work. We had mentioned before that there is a closed link between fractal dimension 


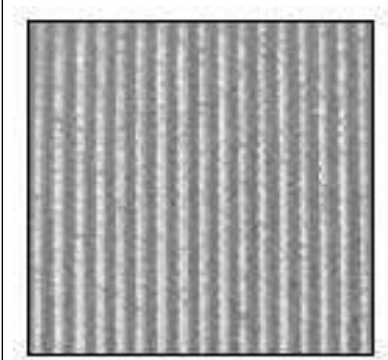

S1

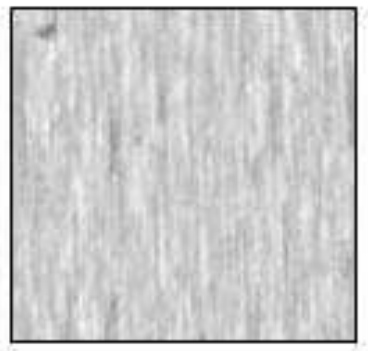

G3

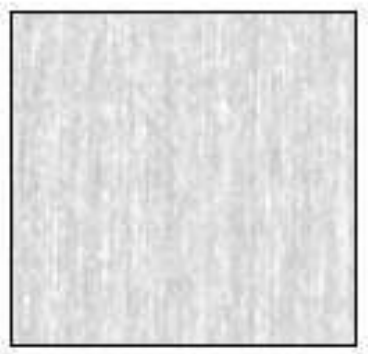

P7

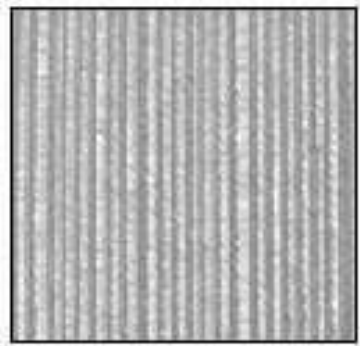

S2

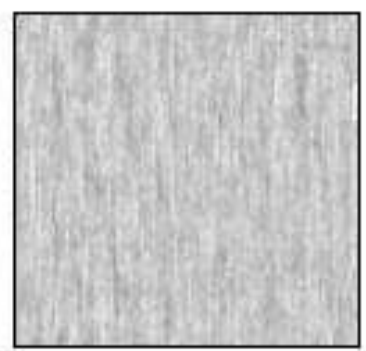

G4

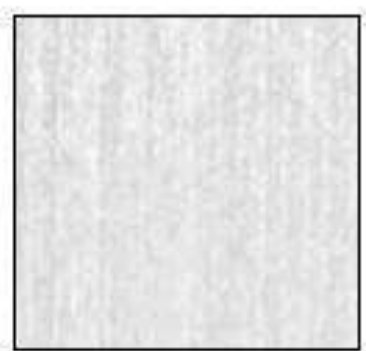

P8

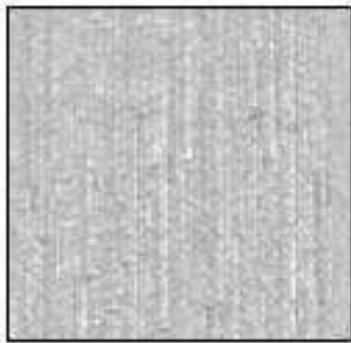

S3

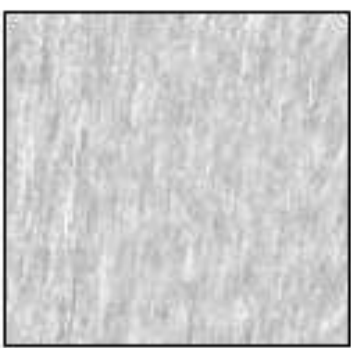

G5

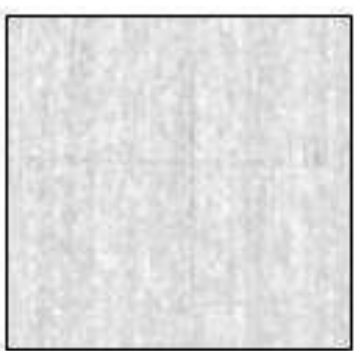

P9

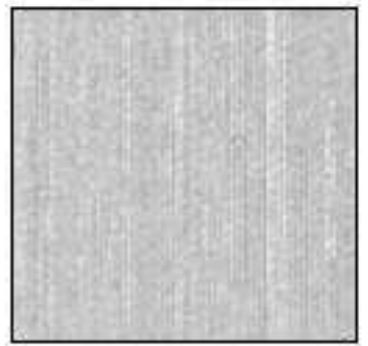

S4

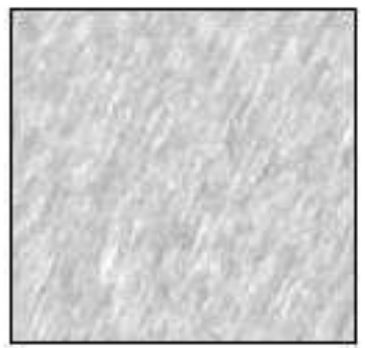

G6

Figure 6: Examples of studied images (magnification x10, coding as stated in Table 1).
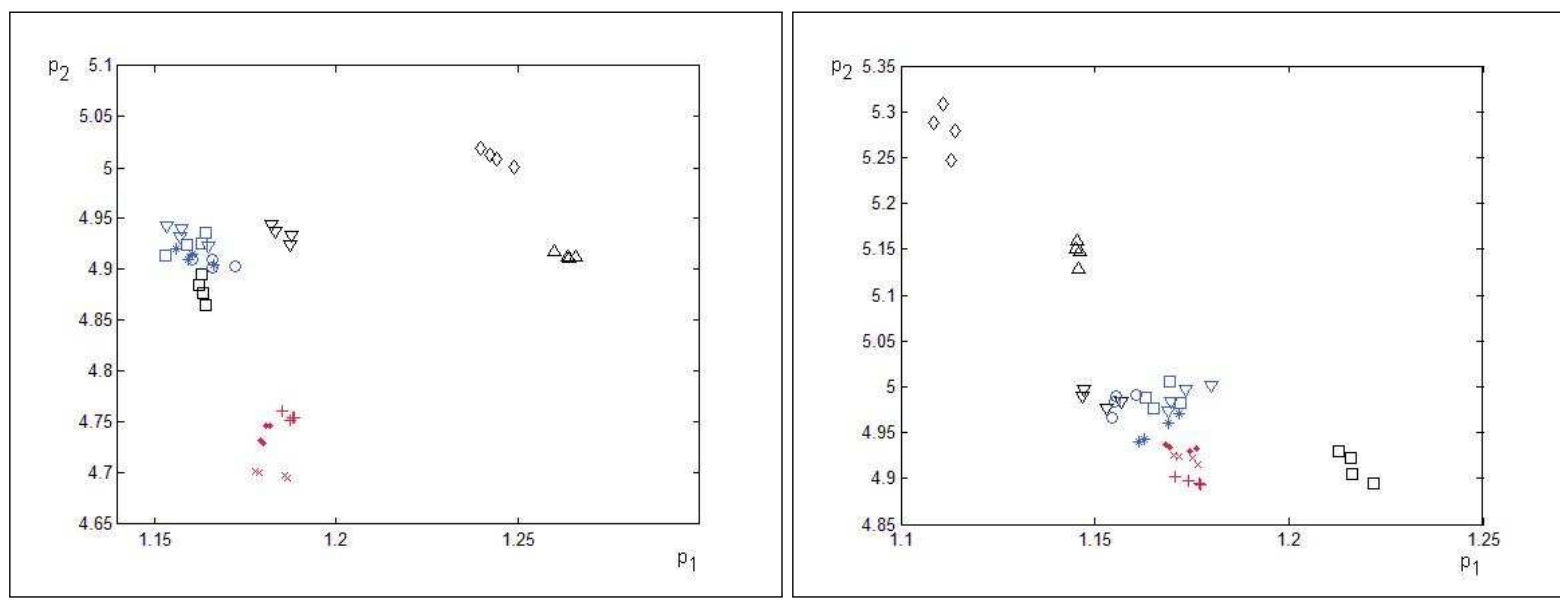

Figure 7: QFP diagrams for parameters $p_{1}-p_{2}$, a. magnification $\mathrm{x} 10$; b. magnification $\mathrm{x} 25$. 
Table 1:

\begin{tabular}{|l|l|l|l|}
\hline $\begin{array}{l}\text { Machining } \\
\text { operation }\end{array}$ & $\begin{array}{l}\text { Roughness } \\
(\mathrm{Ra})\end{array}$ & Code & $\begin{array}{l}\text { Diagram } \\
\text { symbol }\end{array}$ \\
\hline \multirow{4}{*}{ Shaping } & $12.5-6.3$ & $\mathrm{~S} 1$ & Black diamond \\
\cline { 2 - 4 } & $6.3-3.2$ & $\mathrm{~S} 2$ & Black up pointing triangle \\
\cline { 2 - 4 } & $3.2-1.6$ & $\mathrm{~S} 3$ & Black down pointing triangle \\
\cline { 2 - 4 } & $1.6-0.8$ & $\mathrm{~S} 4$ & Black square \\
\hline \multirow{5}{*}{ Grinding } & $3.2-1.6$ & $\mathrm{G} 3$ & Blue down pointing triangle \\
\cline { 2 - 4 } & $1.6-0.8$ & $\mathrm{G} 4$ & Blue square \\
\cline { 2 - 4 } & $0.8-0.4$ & $\mathrm{G} 5$ & Blue circle \\
\cline { 2 - 4 } & $0.4-0.2$ & $\mathrm{G} 6$ & Blue star \\
\hline \multirow{3}{*}{ Polishing } & $0.2-0.1$ & $\mathrm{P} 7$ & Red plus sign \\
\cline { 2 - 4 } & $0.1-0.05$ & $\mathrm{P} 8$ & Red $\mathrm{x}$ \\
\cline { 2 - 4 } & $0.05-0.025$ & $\mathrm{P} 9$ & Red dot \\
\hline
\end{tabular}

and power spectral density parameters, obtained with FFT methods [1] and [2]. The authors had made experiments with the PSD method too, in order to compare the two methods. For the PSD method the same probes were used as in the first case. The obtained results were very close to results obtained with the box counting method. Here an issue to solve is the automated selection of the analysis frequency range. Establishing samples discrimination can be made automatically by a series of clustering methods, like fuzzy c-means or artificial neural networks. The goal of applying clustering methods is to find the cluster center for each sample with known roughness parameter (training phase). After finding the cluster central point this will be used to classify unknown roughness samples (recognition phase). In this phase we will discuss only the training phase.

Regardless what kind of clustering method we use, a good practice is to make the discrimination in successive steps:

1. plot the QFP diagrams for combinations of quasi-fractal parameters taken by two (it is also possible to use higher order QFP spaces, but these can not be properly represented in diagrams);

2. apply a clustering algorithm and find cluster centers;

3. observe which samples are well discriminated, store the cluster centers for these samples and eliminate them from the data set;

4. restart from step 1. with the remaining samples, until all the samples are discriminated.

\section{Fuzzy C-Means Clustering of Quasi-Fractal Parameters}

In order to perform sample discrimination, we choose to use the fuzzy c-means clustering method, which has been implemented in some programming environments as for example in MATLAB. This method does not need large sets of data for training and was suitable for our purpose. In this method each sample is considered to be a part of a cluster, in some degree, defined by a membership degree. This method has been introduced in [13], as an enhancement of existing clustering methods. The method solves the problem of clustering by grouping data sets in multi-dimensional spaces in a number of specified clusters. The method starts with an estimation of the cluster centers marking the central value of each cluster. In the same time, for each sample is assigned a membership degree, which reflects the 
belonging of that sample to certain cluster. The initial cluster center is then successively modified in order to achieve the minimum of an objective function which is the distance of a sample to the center, weighted by the samples membership degree.

We are presenting in the followings the results obtained applying the fuzzy c-means clustering method to the QFP spaces of the described samples. Final clustering is achieved in 4 phases.
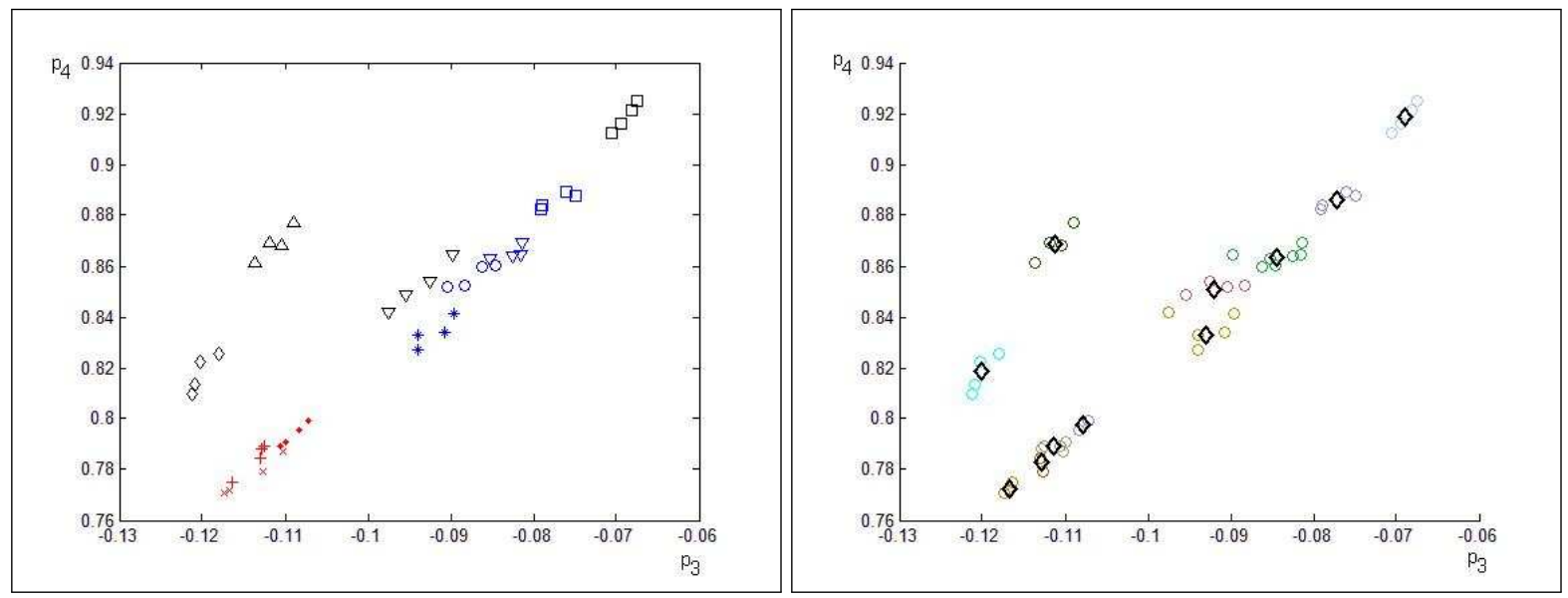

Figure 8: QFP diagram (a) and clustering (b) for phase 1.

The black diamond markers in (b) represent the cluster center and each color represents one cluster.
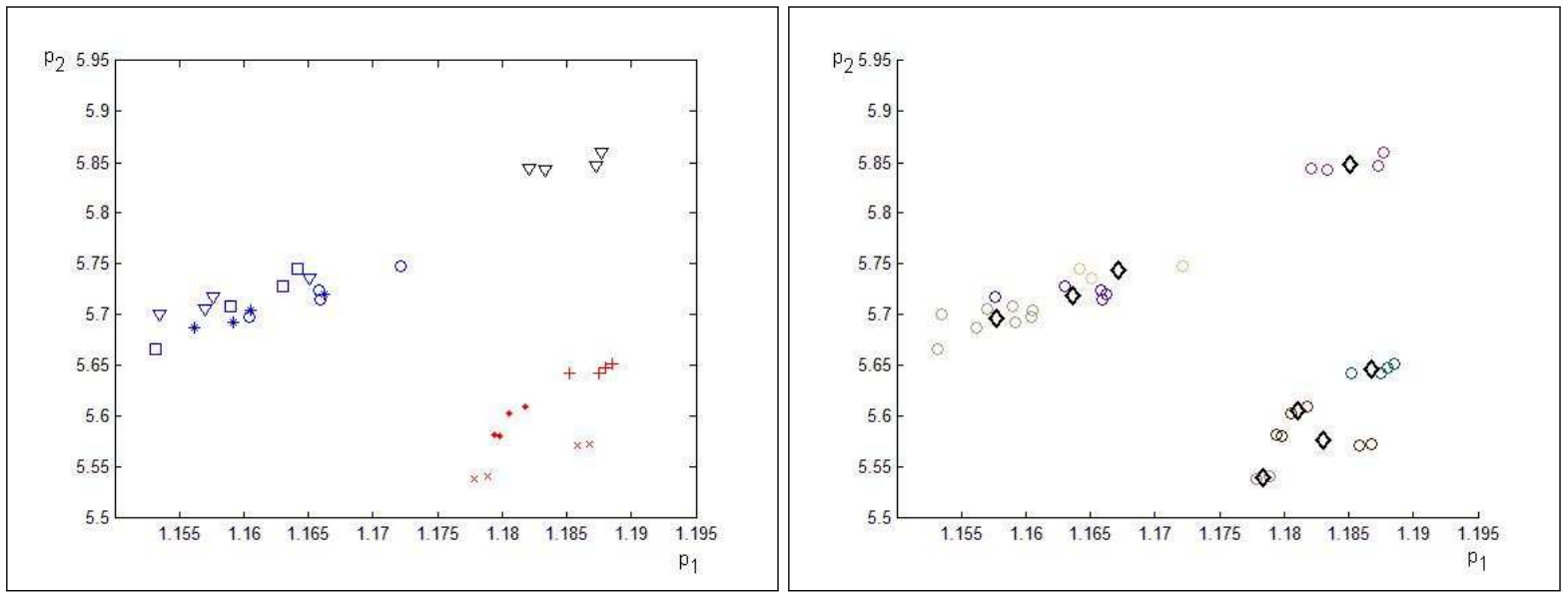

Figure 9: QFP diagram (a) and clustering (b) for phase 2.

The black diamond markers in (b) represent the cluster center.

In the first phase (figure 8) we could find a well discriminated clustering of samples S1, S3, S4, in the second phase (figure 9) we discriminate S2, in the third (figure 10) P1, P2, P3 and G6 and eventually in phase four (figure 11) G3, G4, G5. The found cluster centers will serve to classify any unknown sample later on. In order to increase clustering precision, a combination of different diagrams can also be used.

\section{Conclusions}

In this paper new quasi-fractal parameters $\left(p_{1} \ldots p_{9}\right)$ were defined and a new type of diagram (QFP diagram) was proposed to achieve roughness image recognition. 

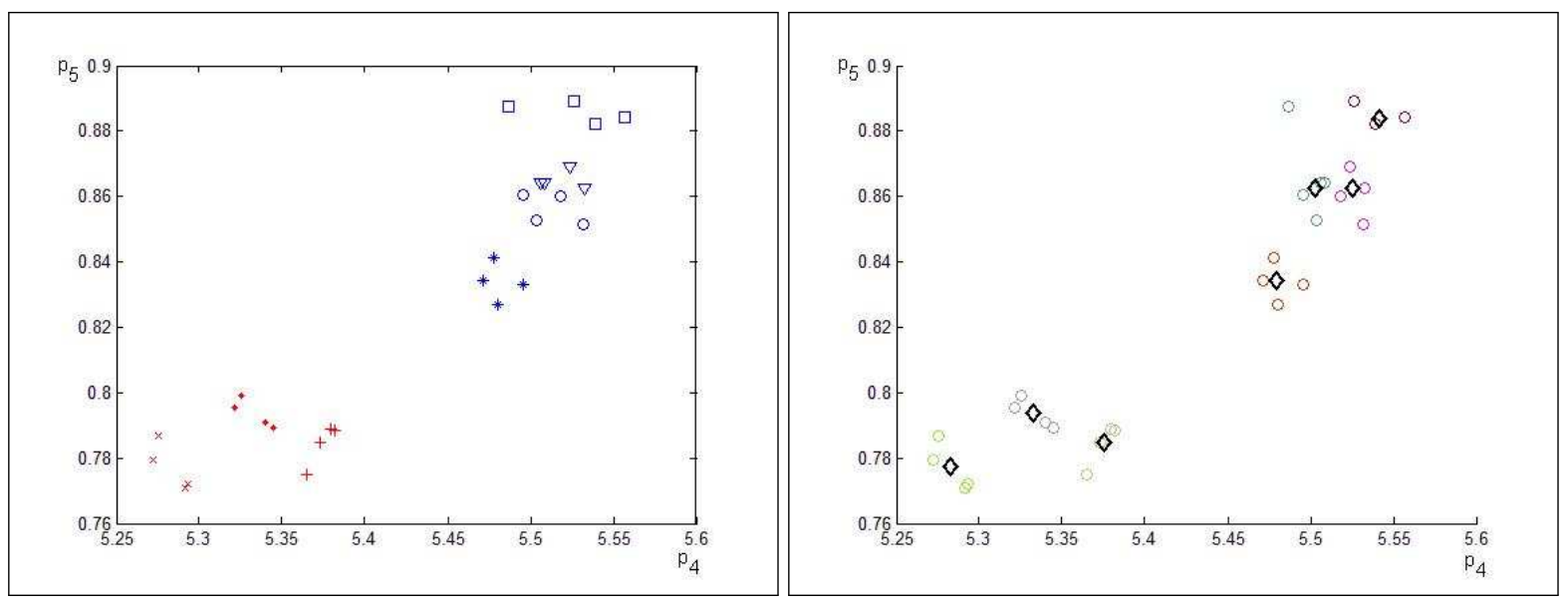

Figure 10: QFP diagram (a) and clustering (b) for phase 3.
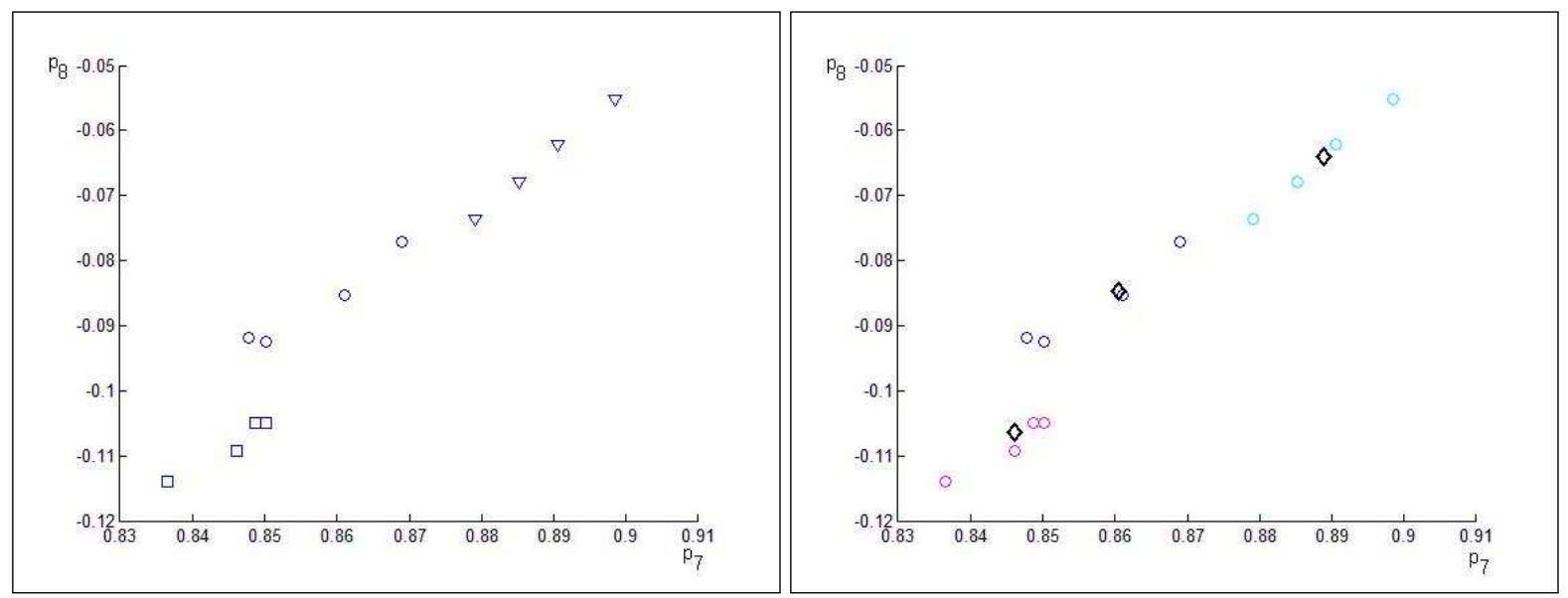

Figure 11: QFP diagram (a) and clustering (b) for phase 4. 
The presented method is not an optimal one since the selection of parameters for the different phases was made by hand, but this selection is needed to be done only once for a group of roughness samples obtained with certain manufacturing methods. The algorithms must also be tested on larger sets of images.

As further developments the selection of box dimension range for BC3D method and frequency range for PSD method will be studied. We will also study the influence of magnification on quasi-fractal parameter discrimination, and a method to find optimal parameter selection.

\section{Bibliography}

[1] M. A. Costa - Fractal Description of Rough Surfaces for Haptic Display - PhD Thesis, Stanford University, 2000.

[2] Z. Jiang; H. Wang; B. Fei - Research into the application of fractal geometry in characterising machined surfaces, International Journal of Machine tools and Manufacture, Elsevier Science Ltd, 2001.

[3] R. K. Leach - NanoSurf IV: traceable measurement of surface texture at the National Physical Laboratory, International Journal of Machine tools and Manufacture, Elsevier Science Ltd, 2001.

[4] B. Y. Lee; Y. S. Tang- Surface roughness inspection by computer vision in turning operations, International Journal of Machine tools and Manufacture, Elsevier Science Ltd, 2001.

[5] D. M. Tsai; S. K. Wu; M.C. Chen- Optimal Gabor filter design for texture segmentation using stochastic optimization, Image and Vision Computing, Elsevier Science, 2000.

[6] S. Baleix, S. Le Roux, G. Bernhart, P. Luors- Surface and image analysis of oxides grown and spalled on heat resistant cast steels exposed on thermal cycles, Ecole des Mines d'Albi-Carmaux, Albi, France, Journal of Materials processing Technology, Elsevier Science BV, 2001.

[7] S. Brinkmann, H. Bodschwinna, H. W. Lemke - Accessing roughness in three-dimensions using Gaussian, regression filtering, University of Hanover, Germany, International Journal of Machine tools and Manufacture, Elsevier Science Ltd, 2001.

[8] S. Lu; Y. Gao; T. Xie; F. Xie; X.Q. Jiang; Z. Li; F. Wang - A novel contact/non-contact hybrid measurement system for surface topography characterization Huazhong, Hong Kong University of science and Technology, Second Research Institute of the Public Security Ministry, China, International Journal of Machine tools andManufacture, Elsevier Science Ltd, 2001.

[9] B. Josso, D. R. Burton, M. J. Lalor - Wavelet strategy for surface roughness analysis and characterisation, Liverpool Jhon Moores University, UK, Computer Methods in Applied Mechanics and Engineering, Elsevier Science, 2001.

[10] K. Yanagi, S. Hara, T. Endoh - Summit identification of anisotropic surface texture and directionality assessment based on asperity tip geometry, Nagaoke University of Technology, Tokyo Institute of Technology, Japan, International Journal of Machine tools and Manufacture, Elsevier Science Ltd, 2001.

[11] H. Zahouani, M. Assoul, R. Vargiolu, T. Mathia - The morphological tree transform of surface motifs. Incidence in tribology, Ecole Centrale de Lion, Universite France Comte, France, International Journal of Machine tools and Manufacture, Elsevier Science Ltd, 2001. 
[12] M. Wieczorowski - Spiral sampling as a fast way of data acquisition in surface topography, International Journal of Machine tools and Manufacture, Elsevier Science Ltd, 2001.

[13] J. C. Bezdec- Pattern Recognition with Fuzzy Objective Function Algorithms, Plenum Press, New York, 1981.

[14] C. Aguilera, M. Ramos, G. Roa- An Automatic Grading System for Panels Surfaces Using Artificial Vision, International Journal of Computers, Communications and Control, Vol. I (2006), No. 2, pp. 15-22, 2006.

\title{
Tiberiu Vesselenyi \\ University of Oradea
}

Universitatii St. 1, 410087, Oradea, Romania

E-mail: tvesselenyi@yahoo.co.uk

Ioan Dzitac

Department of Economics

Agora University of Oradea

Piata Tineretului 8, Oradea 410526, Romania

E-mail: idzitac@univagora.ro

\author{
Simona Dzitac \\ University of Oradea \\ Universitatii St. 1, 410087, Oradea, Romania \\ E-mail: simona.dzitac@gmail.com \\ Victor Vaida \\ University of Oradea \\ Universitatii St. 1, 410087, Oradea, Romania \\ SC Electrocentrale Deva SA \\ Str. Santierului, nr.1, Mintia, Romania \\ E-mail: vaida@termodeva.ro
}

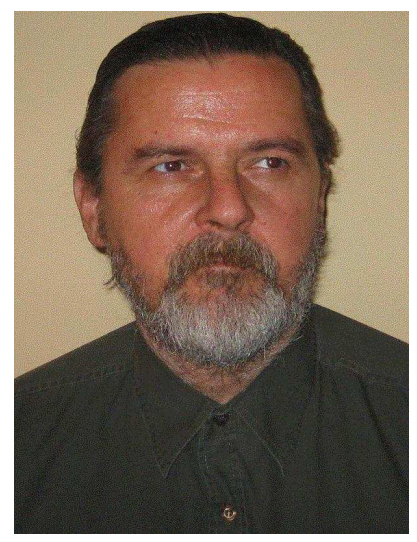

Tiberiu Vesselenyi was born in Oradea, Romania in 1957, he finished the University "Politehnica" from Timişoara in 1983. From 1983 to 1991 he worked at a machine building company in Oradea as designer and CNC programmer. From 1991 till 1994 he was a research engineer at the "Geothermal Energy Research Center" in Oradea and from 1994 till today is assoc. prof. at the University Of Oradea, where he teaches robot and CNC programming. He had earned a PhD in robotics at the University "Politehnica" at Timişoara. He had published over 150 papers in national and international conferences and journals, and is author or coo - author of 4 books. 


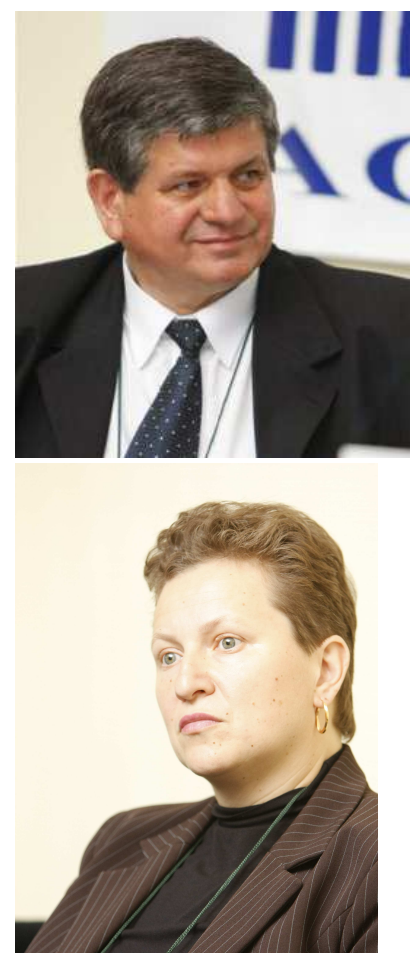

Ioan Dzitac received M. Sc. in Mathematics (1977) and Ph.D in Information Sc. (2002) from "Babes-Bolyai" University of ClujNapoca. At this moment, he is associate professor and head of Economics Department at Agora University, Oradea, Romania. His current research interests include different aspects of Parallel and Distributed Computing, Applied Mathematics and Economic Informatics. He has edited 6 conference proceedings, published 15 books and more than 50 scientific papers in journals and conferences proceedings. He was member of the Program Committee of 27 international conferences.

Simona Dzitac received B.Sc. (2000) and M. Sc. (2001) in Mathematics-Physics, B.Sc. (2005) and M. Sc. (2007) in Energy Engineering from University of Oradea and B.Sc. in Economic Informatics (2007) from University of Craiova, Romania. At this moment, she is $\mathrm{PhD}$ student in Energy Engineering field and researcher at University of Oradea. Her current research interests include Reliability, Applied Mathematics and Computer Science in Engineering fields. She published 5 books and 45 scientific papers in journals and conferences proceedings.

Victor Vaida graduated from the Technical University of Timisoara, Faculty of Electrotechnics, being awarded a B.Sc degree in Electrical Power Engineering in 1968. In 1997 he was awarded a PhD in Technical Sciences by Transilvania University of Brasov. He completed several specialization trainings and courses: in automation, electric and information systems in power plants at Siemens company in Germany (1972 - 1974), in the Quality of Electricity Supply (2007 - 2008) at SIER Bucharest and in business management at the Bucharest Institute of Management (1994). At present, he is the Technical Manager of SC Electrocentrale Deva and an Associate Professor of the University of Oradea, Faculty of Power Engineering. He is also an associate researcher at the Economy and Industry Institute of the Romanian Academy and the President of the Society of Power Engineers of Romania. His research activity includes: 7 specialized books and 160 articles in the power field published in Romania and abroad, 27 contract/grant-based research projects and some other R and D. 